\title{
LES RENCONTRES ANNUELLES DU CETIOM 2000 Paris-Toulouse du 28/11 au 1/12/2000
}

Oléagineux, Corps Gras, Lipides. Volume 7, Numéro 4, 309, Juillet - Août 2000, La filière, aujourd'hui, demain

\section{Tournesol - Paris, mardi 28 novembre 2000}

$(9 \mathrm{~h}-17 \mathrm{~h})$

* Bilan de la campagne tournesol 2000 - Jean Raimbault (Cetiom, Agen)

* Mildiou du tournesol : reconversion, sélection, complémentarité avec la lutte chimique, durabilité des résistances - Étienne Pilorg (Cetiom, Grignon)

* Point des études sur le Gaucho :

- résultats des travaux INRA sur le comportement des abeilles - Axel Decourtye (INRA, Bures-surYvette)

- résultats sur la présence d'imidaclopride dans les plantes - Fabien Lagarde (Cetiom, Grignon)

* Analyses des raisons de l'irrégularité des rendements du tournesol - Luc Champolivier (Cetiom, Grignon)

* Tournesol biologique :

- Cadre réglementaire et perspectives d'évolution du tournesol biologique - (FNAB)

- Avancées techniques dans le domaine de la production agricole - Christophe Bonnemort (Cetiom, Béziers)

* Intérêt nutritionnel de l'introduction du tournesol oléique dans les huiles combinées - $D r$ Bernadette Delplanque (Université d'Orsay)

* Tournesol et biotechnologies : où en sommes-nous ? - Günther Hahne (CNRS, Strasbourg)

\section{Perspectives - Paris, mercredi 29 novembre 2000}

$(9 \mathrm{~h}-17 \mathrm{~h})$

* Compétitivité des oléagineux

- Conséquences du nouveau règlement de Berlin - Olivier de Gasquet (FOP)

- Perspectives d'évolutions des assolements et des revenus agricoles - Jean-Claude Sourie (INRA, Grignon)

- Analyse de l'évolution des surfaces et des rendements à l'échelle régionale - André Pouzet (Cetiom, Paris)

- Analyse prospective de l'évolution de la compétitivité des oléagineux - Clementina Sebillotte (Cetiom, Grignon)

* OGM et expérimentation au champ

- L'expérimentation au champ face au principe de précautions

- Évolution de la réglementation pour la mise en culture des OGM et l'expérimentation - Hervé Reverbori (ministère de l'Agriculture DGAL, Paris) 
- Les différentes approches de l'évaluation des risques environnementaux - Pierre-Henri Gouyon (CNRS, Orsay)

- Impact des colzas tolérants à un herbicide dans les systèmes de culture - Antoine Messéan (Cetiom, Grignon)

Colza - Paris, jeudi 30 novembre 2000

$(9 \mathrm{~h}-17 \mathrm{~h})$

* Bilan de la campagne colza 2000 - Julien Charbonnaud (Cetiom, Ardon)

* Sensibilité des méligèthes aux insecticides - Yannick Ballanger (Cetiom, Grignon)

* Le sclérotinia en 2000 et les résistances à la carbendazime : bilans et conséquences pratiques pour la lutte - Marie-Jo Kaczmar (SRPV, Franche-Comté), Annette Penaud (Cetiom, Grignon)

* Valeur des différents critères utilisables pour caractériser les risques de pertes en nitrates : intérêts et limites des indicateurs « azote » - Raymond Reau (CETIOM, Grignon)

* Le point sur les nouveaux hybrides - Xavier Pinochet (Cetiom, Grignon)

* Le développement du colza en Pologne - Iwona Bartkowiak-Broda (IHAR, Poznan)

* La trituration par pression-extrusion bi-vis : une technologie innovante - Pierre Burghart (Cetiom, Pessac)

Soja - Toulouse, vendredi $1^{\mathrm{er}}$ décembre 2000

$(14 \mathrm{~h}-17 \mathrm{~h})$

* Bilan de la campagne soja 2000 - Vincent Lecomte (Cetiom, Baziège)

* Maîtrise de la teneur en protéines par la conduite de culture - Philippe Burger (INRA, Toulouse)

* Étude des transferts d'azote dans les plantes au cours du remplissage chez le soja - David Grandgirard (INRA, Dijon)

* Les valorisations de nouvelles filières

- Soja biologique

- Organisation de la production agricole - Salvador Ferre (Agribio Union)

- La filière de transformation et son organisation - Bernard Storup (Nutrition et Soja)

- La filière « Soja de pays » - Xavier Dorchies (Sofiproteol)

Paris : Paris-Expo - Porte de Versailles 75015 - Porte A - Hall 7 - Mezzanine 2 - Salle 725 (Amphi Lyra)

Toulouse : INRA - Auzeville, Salle de réunion des services généraux - Chemin de Borde-Rouge - 31326 Castanet - Tolosan

[Des plans d'accès sont fournis avec l'inscription]

Contact : Josiane Lochouarn

Fax : 0130799590 